\title{
Optimierte Modelle der Friedenssicherung durch internationale Rechtsprechung und Rechtsdurchsetzung
}

\author{
Dr. Erwin Müller/Dr. Patricia \\ Schneider, Institut für Frie- \\ densforschung und Sicher- \\ heitspolitik an der Universität \\ Hamburg.
}

\section{Einleitung}

Gerade in (völker-)rechtlich gesehen »finsteren Zeiten «, in denen missionarisch inspirierte oder kaltblütig kalkulie-

rende Machthaber der Durchsetzung ihrer Interessen freien Lauf lassen, ist es besonders wichtig - allen Widrigkeiten zum Trotz - an dem einmal erreichten Stand des langwierigen »Prozesses der Zivilisation « festzuhalten, insbesondere, was die »Zivilisierung des Konfliktaustrags« betrifft. Dem wiederbelebten »Faustrecht « ist das Prinzip der Herrschaft oder zumindest des Primats des Rechts entgegenzusetzen. Wenn es spezifisch um den Frieden als Wert geht, so lässt sich an die Maxime »Frieden durch Recht « im übergeordneten Sinne von »Frieden durch Gerechtigkeit« appellieren.

Ein zentrales Element des Sprungs aus dem Reich der Barbarei mit dem »Recht« des Stärkeren in das Reich der Zivilisation, in dem Recht vor Macht geht, verkörpert die internationale (Schieds-)Gerichtsbarkeit, im Bedarfsfall ergänzt von anderen Formen der friedlichen Streitbeilegung.

Diese institutionellen Arrangements unterteilen sich systematisch in Mechanismen der Streit-Erledigung bzw. StreitBeilegung als Oberbegriff und - als Unterkategorien - in die Instrumente der Streit-Schlichtung (in Form des Vergleichs) und der Streit-Entscheidung in Gestalt von Schiedsgerichtsbarkeit (Arbitration) und Gerichtsbarkeit (Jurisdiktion): Schlichtung bzw. Vergleich produzieren Empfehlungen für die Bereinigung einer Streitigkeit, (Schieds-) Gerichtsverfahren schließen sie durch einen autoritativen (Urteils-)Spruch ab.

Die unschätzbaren Vorzüge der arbitralen und jurisdiktionellen Streitentscheidung bestehen in ihren folgenden Charakteristika:

1. Der relevanteste Aspekt dürfte in der Tatsache liegen, dass vor Gericht jedes Machtgefälle zwischen den Streitparteien dadurch aufgehoben ist, dass beide Seiten idealiter absolut gleich behandelt werden und nur ihre jeweiligen Rechte zählen, so dass die reale Chance zu einer fairen und gerechten Streiterledigung besteht.

2. Ein formalisiertes und versachlichtes Verfahren vor einer unparteiischen Instanz mäßigt die Emotionen der Parteien, wirkt deeskalierend und inspiriert sie zu einem »zivilisierten« Auftreten vor der Institution. Es findet eine Konflikt-Transformation vom politischen Konflikt zum Rechts-Streit statt. Diese »Entpolitisierung « reduziert den Grad der Konfrontation, da nur seriöse (Rechts-) Argumente zählen und aggressiv-ideologische Bekundungen beim Entscheidungsgremium nur Befremden hervorrufen und im Zweifelsfalle eher abträglich sind.
»Iustitia fundamentum pacis«

3. Die in einem in dieser Form ausgetragenen Rechtsstreit unterliegende Partei kann zur »Gesichtswahrung « darauf hinweisen, dass eine »übergeordnete « Instanz die entsprechende ungünstige Entscheidung gefällt hat, diese also nicht unter Druck der anderen Partei hingenommen wird, sondern aus »Achtung vor dem Recht« im Sinne einer von ihm verkörperten »höheren Gewalt«.

Diesen mannigfaltigen Vorzügen zum Trotz wird vom System der internationalen (Schieds-)Gerichtsbarkeit von der Staatenwelt nach wie vor nur höchst unzulänglich Gebrauch gemacht. Das hängt nicht zuletzt mit ihren systemaren Defiziten zusammen, die im Folgenden herausgearbeitet werden. Auf der Grundlage dieser Analyse werden in der Konsequenz Reformvorschläge in Gestalt von Alternativmodellen internationaler (Schieds-)Gerichtsbarkeit vorgestellt, die den erkannten Defiziten abhelfen sollen.

\section{Kardinaldefizite internationaler (Schieds-)Gerichts- barkeit}

Trotz aller Erfolge, die die internationale (Schieds-)Gerichtsbarkeit bisher für sich verbuchen konnte, bleibt doch die Tatsache bestehen, dass die Staaten nur eine insuffiziente Bereitschaft aufweisen, die Gerichtsbarkeit etwa des IGH anzuerkennen bzw. sich an diesen zu wenden. So konnten zahlreiche internationale Konflikte lange Zeit nicht gelöst und Gewaltanwendung vielfach nicht verhindert werden: Das Prinzip der völligen Freiwilligkeit der Staaten bei der Nutzung der internationalen (Schieds-)Gerichtsbarkeit führt nicht zu angemessenen Resultaten der friedlichen Streitbeilegung und wird dem Gewaltverbot somit nicht gerecht.

Das erste Haupt- oder Kardinaldefizit besteht mithin in der nicht-obligatorischen Ausgestaltung internationaler (Schieds-)Gerichtsbarkeit: Bei einseitiger Klage besteht unter diesen Prämissen für die andere Partei kein Einlassungszwang, mit der Folge, dass sie sich einem Verfahren entziehen kann. Ferner verweist die empirische Untersuchung des internationalen Jurisdiktions- und Arbitrationssystems auf gravierende Defizite in der (zwangsmittelbewehrten) $U r$ teilsvollstreckung gegen Widerstreben der aus dem Urteil verpflichteten Partei: Autoritative Entscheidungen ohne eine Autorität (und die Instrumente) zu ihrer Durchsetzung erweisen nur die Ohnmacht der Entscheidungsinstanz und unterminieren das in sie gesetzte Vertrauen. Letztendlich erhebt sich für potenzielle Kläger, die einen Streit mit Hilfe solcher Institutionen friedlich (und möglichst $\mathrm{zu}$ eigenen Gunsten) aus der Welt schaffen wollen, die Frage, weshalb sie sich die Mühe machen sollten, ihr Recht vor einer Instanz 
zu suchen, die es ihnen im Zweifelsfalle nicht verschaffen kann.

Als weiteres Problem ist - insbesondere im Falle des IGH die historisch überholte, überzogene Einschränkung der Parteifähigkeit $\mathrm{zu}$ konstatieren.

Aus diesen Mängeln erwachsen die im Folgenden benannten Anforderungen an eine Systemreform.

\section{Minimaldesiderate effektiver (Schieds-)Gerichts- barkeit}

Wie erläutert, garantiert die Existenz eines Gerichts als solche keineswegs den Zugang aller Gruppen oder Institutionen, die des Rechtsschutzes bedürfen. Ohne Einlassungszwang greift seine Jurisdiktion nicht, ohne Urteilsdurchsetzungskompetenz besteht die Gefahr der Missachtung seiner Entscheidungen. Aus diesen Defiziten ergeben sich im Sinne ihrer Behebung einige Minimaldesiderate effektiver (Schieds-)Gerichtsbarkeit.

\subsection{Parteifähigkeit}

Die Parteifähigkeit wäre über Staaten hinaus auf internationale Organisationen und - wo sinnvoll - auf substaatliche Gruppen, insbes. Minderheiten, zu erstrecken.

Desgleichen könnte darüber nachgedacht werden, ob man den Zugang zum IGH im Bereich der Rechtsgutachten (advisory opinions) für jene internationalen Organisationen öffnet, die in den internationalen Beziehungen neben den souveränen Staaten eine immer größere Rolle spielen und bislang entsprechende Rechte nicht besitzen.

Auch dem VN-Generalsekretär könnte die Antragsbefugnis für Gutachtenanfragen eingeräumt werden, insbes. zur Klärung von institutionellen Streitfragen, die rechtliche Aspekte besitzen, sowie zur Klärung der rechtlichen Seite von Streitfällen, in denen er um Vermittlung oder gute Dienste gebeten worden ist. Eine entsprechende Befugnis der im IGH-Statut befindlichen Staaten sollte ebenfalls erwogen werden.

Was die Parteifähigkeit in streitigen Verfahren anbelangt, so werden sich internationale Organisationen ähnlich wie Staaten via Eigenklage i.d.R. selbst behelfen können. Anders kann es sich möglicherweise im Fall von substaatlichen Entitäten wie insbesondere nationalen Minderheiten verhalten, denen ein Klagerecht aus dem naheliegenden Grunde eingeräumt werden sollte, dass die aktuelle Konfliktlandschaft sich eher durch binnenstaatliche Auseinandersetzungen auszeichnet als durch zwischenstaatliche Konfrontationen. Hier können sich Situationen ergeben, in denen sich eine solche Gruppe durch ihre Staatsgewalt an der Äußerung ihres Willens gehindert sieht.

Um einer diskriminierten bzw. bedrängten Minderheit die Chance zu gewähren, den Zugang zu einem internationalen Gericht zu finden, selbst wenn sie sich aus Gründen verschärfter Repression nicht selbst artikulieren kann (oder weil sie vor Gericht keine Parteifähigkeit besitzt), ist die Implementation von variablen (»Stellvertreter«-)Optionen von
Nöten. Terminologisch könnten sie als »Patronagelösung", als »Ombudsmannversion" oder »Advokatenvariante" bezeichnet werden. Ihnen gemeinsam wäre die Eigenschaft, dass eine Institution subsidiär tätig würde, wenn der unmittelbar rechtsschutzbedürftigen Gruppe die Hände gebunden sind, entweder auf Grund ihrer Anrufung oder aus eigener Initiative (s. Abb. 2).

Als Patron, also Schirmherr oder Schutzherr bzw. Protektor (»Schutzmacht« im Sinne der völkerrechtlichen Protektion, nicht des bevormundenden Protektorats) käme ein Staat in Frage, der zu einer Volksgruppe in besonders enger Beziehung steht und ihr Vertrauen genießt, etwa aus Gründen einer ethnischen Affinität. Die Rechtsgrundlage für die Patronagekompetenz wäre durch völkerrechtlichen Vertrag zwischen dem Patronagestaat und dem Heimatstaat einer Volksgruppe zu schaffen, der insbesondere die Gerichtsbarkeit etwa des IGH in dieser Angelegenheit herstellt. In diesem Vertrag wären auch die Rechte der Volksgruppe festzulegen, die vom Protektor notfalls eingeklagt werden können. Eine Rechtsverletzung würde diesen Staat im Prinzip dann selbst betreffen, da die Einhaltung eines bestimmten Rechtsstandards ihm selbst gegenüber zugesagt wurde. Er wäre somit als Partei anzusehen, da die Verletzung der Volksgruppenrechte als Verletzung seiner eigenen Rechte einzustufen wäre. Ferner soll die gewählte Bezeichnung »Patron« oder »Protektor« auch signalisieren, dass er sich durchaus als Interessenvertreter seiner Protegés verstehen darf, ohne dass er allerdings von ihnen unbedingt ein formelles Mandat erhalten hätte. Die Tatsache, dass dem Patronagestaat aus alledem kein Recht zur Gewaltanwendung zu Gunsten seiner Schützlinge erwächst, versteht sich von selbst.

Wo es nicht um bestimmte Minderheitenrechte als Kollektivrechte, sondern um die individuellen Menschenrechte geht, wäre (nach dem Muster der regionalen Europäischen bzw. Amerikanischen Menschenrechtskonvention) ein generelles Klagerecht für alle Vertragsstaaten einer entsprechenden globalen Konvention vorzusehen. In diesem Kontext sollte die Errichtung eines Welt-Menschenrechtsgerichtshofs ins Auge gefasst werden, zu dem auch Individuen und zivilgesellschaftliche Gruppen Zugang haben müssten.

Im Rahmen des Minderheitenschutzes erscheint eine Lösung, die sich als Ombudsmannprinzip bezeichnen lässt, günstiger als die Patronagelösung, da in diesem Falle die Objektivität der handelnden Instanz gewährleistet wäre (im Gegensatz zur durchaus zulässigen Subjektivität des Protektorstaates). Sie wäre insofern weder Partei noch parteiisch, als sie zum einen von einer internationalen Einrichtung oder durch einen multilateralen Vertrag eingesetzt und mit ihren Kompetenzen ausgestattet wäre, zum anderen - dem bekannten Ombudsmann-Prinzip entsprechend - keiner Partei oder Gruppe bzw. ihren subjektiven Interessen verpflichtet wäre, sondern nur einer objektiven Rechtsordnung bzw. dem öffentlichen Interesse. Das Modell lehnt sich - neben dem Ombudsmann-Prinzip - an die Charakteristika internationaler (regionaler) Menschenrechtskommissionen wie auch der EU-Kommission an, die allesamt zu einer objektiven Prüfung (»sine ira et studio«) der ihnen zu Gehör kommenden potenziellen Verstöße gegen die von ihnen zu wahrende Rechtsordnung verpflichtet sind, bevor sie in einer Sache tätig werden. Andererseits sind sie aber auch dazu angehalten, 
bei Feststellung einer Rechtsverletzung eines Vertragsstaates gegenüber Dritten, die in ihren Kompetenzbereich fällt, tätig zu werden. Auch die hier angesprochene Instanz tut dies nicht als Anwalt einer Partei, sondern nur als Anwalt (oder Hüter) des Rechts und erfüllt somit eine öffentliche Funktion im Rahmen einer internationalen Rechtsgemeinschaft im Sinne eines Vertreters des öffentlichen Interesses. (In diesem Sinne könnte auch von einer Advokatenvariante die Rede sein.)

Idealerweise sollte diese Institution oder Behörde nicht nur auf Antrag bzw. Anrufung durch eine sich in ihren Rechten verletzt wähnende Partei tätig werden können, sondern auch aus eigener Initiative, d.h. von Amts wegen. Diese Regelung ergäbe in der Konsequenz ein Recht zur (Rechts-) »Aufsichtsklage « (ähnlich dem der EU-Kommission). In ersterem Falle würde eine objektive und unparteiische Prüfung der Zulässigkeit und Begründetheit einer Beschwerde erfolgen. Beschwerdevoraussetzung wäre im Falle nationaler Minderheiten die Erschöpfung des innerstaatlichen Rechtsweges oder seine Nichtexistenz (s. Abb. 2).

Bringt eine solche Institution eine Sache vor Gericht, so ist sie als unparteiischer Vertreter des öffentlichen Interesses, der einen objektiven Standpunkt verficht, nicht als Partei im herkömmlichen Sinne zu begreifen, obwohl sie alle entsprechenden Rechte wahrnimmt.

Vor Beschreitung des Klageweges sollte sie allerdings versuchen, mit dem betroffenen Staat eine außergerichtliche Lösung des Problems zu finden, was angesichts einer Klageandrohung, deren Abwendung Zugeständnisse attraktiver macht, vielfach gelingen dürfte.

\subsection{Obligatorium}

Um die Funktionsfähigkeit des Gerichtsmechanismus zu garantieren, bedarf es der Einführung einer obligatorischen (Schieds-)Gerichtsbarkeit, die zu einem Einlassungszwang für widerspenstige Parteien führt.

Als Strategien zur Erreichung dieses Ziels bieten sich u.a. an:

- Die zielführende Revision des IGH-Statuts bzw. der Charta der Vereinten Nationen.

- Der Abschluss eines globalen Schiedsvertrags mit entsprechendem Inhalt.

- Die Akzeptanz obligatorischer Gerichtsbarkeit über die Selbstverpflichtung möglichst aller Staaten gemäß der Fakultativklausel des IGH-Statuts unter Verzicht auf die Reziprozitätsklausel und Vorbehalte.

- Die Ermächtigung des Sicherheitsrats der Vereinten Nationen, bei friedensgefährdenden Streitigkeiten die Beteiligten auf Antrag einer Partei oder von sich aus zum Gang vor den IGH oder zur Einleitung eines Schiedsverfahrens zu verpflichten, falls sie seine Empfehlungen zur Konfliktregelung nicht akzeptieren oder ein (fakultativ) von ihm vorgängig verfügtes Schlichtungsverfahren (»Schlichtung auf Anordnung«) ergebnislos verläuft.

\subsection{Urteilsvollstreckung}

Um die Urteilsdurchsetzung zu gewährleisten, sind die bereits angesprochenen Durchsetzungskapazitäten bereitzustel- len, ungeachtet der Tatsache, dass Rechtsgehorsam vielfach auch ohne Sanktionsdrohung geleistet wird.

Zur Durchsetzung des Rechts bedarf es angemessener Fähigkeiten, um die »immoral minority « zur Räson bringen und zur Rechtstreue anhalten zu können, also einer Instanz mit Zwangskompetenz und Zwangsinstrumenten.

Dementsprechend könnte dem Sicherheitsrat der Vereinten Nationen im Gegensatz zur heutigen Ermessensregelung die Pflicht auferlegt werden, Urteilen mittels geeigneter und angemessener Maßnahmen selbständig Geltung zu verschaffen. Um das Gremium an seiner Pflichterfüllung nicht $\mathrm{zu}$ hindern, wäre zumindest in diesen Fällen das Vetorecht zu eliminieren.

In einer weiteren Variante handelt der Sicherheitsrat auf Anordnung: Der IGH könnte bei Nichtbefolgung des Urteils nach einer Frist die Urteilsvollstreckung selbst anordnen und damit den Sicherheitsrat zum Tätigwerden verpflichten. In beiden Fällen erfolgt die Exekution der Gerichtsentscheidung auf Antrag der obsiegenden Partei, die einen vollstreckbaren Rechtstitel präsentieren kann.

Im Regelfall wird sich die Anwendung ökonomischer Maßnahmen wie etwa die Beschlagnahme von Auslands-Bankguthaben und anderen Vermögenswerten als ausreichend erweisen. In besonders gravierenden Fällen kann sich die Ausübung unmittelbaren physischen Zwangs gemäß den Prinzipien der Verhältnismäßigkeit jedoch als unumgänglich herausstellen. Der weitreichenden Konsequenzen eines solchen Vorgehens wegen dürfte es sich empfehlen, es auf Fälle zu beschränken, in denen der Urteilsungehorsam gleichzeitig einen Friedensbruch i.S.d. Charta der Vereinten Nationen darstellt. Im Kontext der Zwangsvollstreckung ist auch die Möglichkeit $\mathrm{zu}$ prüfen, den u.U. blockierten Sicherheitsrat zu umgehen, indem erstens die Rolle des Generalsekretärs so aufzuwerten ist, dass ihm diese Aufgabe pflichtgemäß obliegt. Entweder agiert er (im Extremfall) mit VN-Truppen unter seinem Kommando oder er gewinnt VN-Mitglieder für diese Aufgabe, die jedoch nach seinen Richtlinien und unter seiner Rechtsaufsicht tätig werden.

Ein »demokratischeres « Element könnte hingegen durch die Einbeziehung der Generalversammlung eingeführt werden: Bei Nichtbefolgung eines Urteils könnten Durchsetzungsmaßnahmen beschlossen werden, die somit breitestmögliche Legitimationsbasis erhielten. Die Truppen könnten sich (falls keine eigenständigen VN-Truppen gebildet werden) aus den maßnahmenbeschließenden Staaten rekrutieren. Die operative Umsetzung des Beschlusses ist wiederum Aufgabe des Generalsekretär (s.o.). Problematisch sind allerdings die Schwerfälligkeit des Gremiums sowie der bisher reine Empfehlungscharakter von GeneralversammlungsResolutionen.

In Fällen völkerrechtlicher Delikte, die eine Wiedergutmachung in Form einer materiellen Entschädigung verlangen, könnte ein gewichtiger Anreiz zur Urteilsbefolgung durch die vorherige Erbringung einer Sicherheitsleistung (z.B. einer Pfandhinterlegung) geschaffen werden, die dem »Streitwert « entspricht. Sie fällt bei Nichtbefolgung eines Urteils zugunsten des Klägers diesem zu und fungiert somit als Garantie für die Erfüllung seiner Ansprüche. In anders gelagerten Fällen 
dient sie als Kompensation einer pflichtwidrig verweigerten Leistung beliebiger Art.

Die Urteilsvollstreckung durch Exekutivbehörden - ob gerichtlich angeordnet oder nicht - bedarf zumindest der Rechtsaufsicht durch die judikative Instanz, deren korrigierende Eingriffe etwa eine Verletzung des Verhältnismäßigkeitsprinzips oder eine Ungleichbehandlung unterbinden könnten.

Die Einräumung einer zusätzlichen Fachaufsichtskompetenz wäre unter der Prämisse zu erwägen, dass nicht (nur) die Rechtmäßigkeit von Maßnahmen fraglich wäre, sondern (auch) ihre Zweckmäßigkeit, wenn beispielsweise der Weltsicherheitsrat sich weigert, angemessene Schritte zu unternehmen.

Ordnet das Gericht selbst Zwangsmaßnahmen an, so sollte es optimalerweise auch Herr des Verfahrens bleiben und die Operationen der Exekutivinstanz autoritativ dirigieren können. Ähnliche Mechanismen wären auch für die Vollstreckung der Entscheidungen sonstiger Gerichte wie Schiedsgerichte vorzusehen. Hilfreich wäre ferner die Einrichtung eines compliance observation office im Rahmen der VN, welches die Einhaltung der Urteile des IGH und anderer internationaler Gerichte bzw. Schiedsgerichte beobachtet. Regelmäßige Berichte über die Befolgung von Urteilen und Anordnungen würden der Staatengemeinschaft und der kritischen Weltöffentlichkeit den bisher fehlenden Einblick eröffnen sowie die Bekanntheit dieser Instrumente steigern und ihre Nutzung fördern. $\mathrm{Zu}$ hoffen wäre, dass diese Transparenz einen hohen Erwartungsdruck erzeugt, der jede Missachtung von Gerichtsurteilen als einen empfindlichen Imageverlust erscheinen lässt.

\section{Abb. 1: Spektrum der Optionen zur Urteilsvollstreckung}

1. Sicherheitsrat handelt selbständig (auf Antrag einer Partei)

2. Generalversammlung handelt selbständig (auf Antrag einer Partei)

3. Sicherheitsrat handelt auf Anordnung des IGH

4. wie 3., allerdings nicht Vollstreckung durch Sicherheitsrat, sondern

durch Generalsekretär $\rightarrow$ a) Selbstvornahme

$\rightarrow \quad$ b) Delegation

5. Anreize zur Urteilserfüllung: Sicherheitsleistung, z.B. durch Pfandhinterlegung

6. Vollstreckung nicht nur von Urteilen des IGH, sondern auch der Urteile anderer internationaler Gerichte und Schiedsgerichte (nach Urteilsbestätigung durch den IGH).

\section{Optimierte Modelle Internationaler (Schieds-) Gerichtsbarkeit}

Ein effizientes Modell ohne jede Realisierungschance würde im Bereich des Utopischen bleiben, ein realisierbares ineffizientes Modell wäre den Aufwand für seinen Entwurf nicht wert. Es kommt also entscheidend darauf an, den beiden gegensätzlichen Kriterien zugleich gerecht $\mathrm{zu}$ werden, d.h. bei der Modellbildung die die Realisierbarkeit bestimmende Bereitschaft der Staaten zur Einschränkung ihrer souveränen Handlungsfreiheit ebenso ins Kalkül zu ziehen wie die Minimalkriterien effizienter Mechanismen: Beide Größen verhalten sich i.d.R. umgekehrt proportional bzw. negativ reziprok im Sinne einer negativen Korrelation. Je mehr die eine zunimmt, desto mehr nimmt die andere ab. Somit stellt sich eine klassische Optimierungs-Aufgabe, die die beiden notwendigen Bedingungen (Effizienz und Realisierbarkeit), die aber nur gemeinsam hinreichend sind, zur Konvergenz bringt, d.h. objektives Effizienz-Minimum (Nutzen) und subjektives Souveränitätsverzichts-Maximum (Kosten) miteinander vereinbar macht.

Im Folgenden werden optimierte Reformmodelle für die internationale Streitbeilegung mit dem Schwerpunkt auf Gerichtsbarkeit und Schiedsgerichtsbarkeit vorgestellt, die unterschiedliche Komplexitätsgrade aufweisen und fallweise auch außergerichtliche Methoden als Elemente des Gesamtprozesses einbeziehen. Es ergeben sich somit Basismodelle und komplexe Modellvarianten, die darauf aufbauen.

\subsection{Basismodelle}

Im Blickpunkt dieser Rubrik stehen zwei klassische Modelle, die i.d.R. alternativ und je für sich zum Zuge kommen können: Schiedsgerichtsbarkeit und Gerichtsbarkeit, die sich in mancher Hinsicht von einander abheben. Ein Gericht ist eine existente Institution und braucht daher nicht erst im Streitfalle gebildet zu werden. Die Rechtsgrundlage für seine Urteile ist wie seine Verfahrensordnung bereits fixiert. Verfahren wie Urteilsverkündung sind i.d.R. öffentlich. Die Schiedsgerichtsbarkeit weist im Vergleich dazu eine Reihe von Charakteristika auf, die souveränitätsbewussten Staaten eigentlich entgegenkommen müssten. Diese Eigenschaften könnten optimiert werden, indem das Spektrum der ModellVarianten, dessen Breite die des Gerichtsmodells bei weitem übertrifft, weiter ausdifferenziert wird. Die unvergleichliche Flexibilität des Schiedsgerichtsprinzips gestattet ein solches Vorgehen, ohne dass das Prinzip Gefahr läuft, überdehnt zu werden. $\mathrm{Zu}$ den Kennzeichen und deren Variabilität im einzelnen:

1. Die Wahl der Richter, d.h. die Besetzung der Richterbank, obliegt im Prinzip den Parteien selbst, die auch die Grenzen der dem Schiedsgericht zuerkannten Rechte und Kompetenzen abstecken. Je nach dem Grad der Bereitschaft der Staaten zum selbstgewählten Verzicht auf ihre Einflusschancen auf die Bestimmung des i.d.R. dreioder fünfköpfigen Schiedskollegiums sind verschiedene Varianten der Richterherkunft realisierbar:

- Anzahl und Anteil der von den Parteien einseitig ernannten Richter:
a) 4 von $5(80 \%)$
b) 2 von $3(67 \%)$
c) 2 von $5(40 \%)$

- Vice versa: Anzahl und Anteil der »neutralen« Richter, die von den Parteien oder von den von ihnen einseitig ernannten Richtern einvernehmlich bestellt werden:
a) 1 von $5(20 \%)$
b) 1 von $3(33 \%)$
c) 3 von $5(60 \%)$ 
- Anzahl und Anteil der Schiedsrichter, die die Staatsbürgerschaft der Streitparteien besitzen dürfen:
a) 4 von $5(80 \%)$
b) 2 von $3(67 \%)$
c) 2 von $5(40 \%)$
d) $0 \quad(0 \%)$

Wie ersichtlich, nimmt in allen drei Rubriken der Parteieneinfluss bzw. der Einfluss der einzelnen Partei von a) nach c) bzw. d) ab, immer unter der verbreiteten Annahme, dass die von einer Partei ernannten Personen bzw. ihre Staatsangehörigen dazu tendieren, deren Interessen bevorzugt $\mathrm{zu}$ berücksichtigen. Werden alle »neutralen « Schiedsrichter unmittelbar von einer Drittpartei, etwa einer Schiedsorganisation, bestimmt, so nähert sich der Mechanismus an den der internationalen Gerichtsbarkeit an. Dies gilt umso mehr, wenn diese Organisation auch noch das Völkerrecht als Urteilsgrundlage vorgibt und die Verfahrensordnung festlegt. Dies führt zu der kuriosen Lage, dass der Parteieneinfluss auf die personelle Besetzung von Kammern internationaler Gerichtshöfe (IGH und ISGH) im Vergleich dazu größer ausfällt. Wenn die Institution der Schiedsgerichtsbarkeit ihre unverwechselbaren Charakteristika bewahren soll, dann ist die Akzeptanz einer mehr oder minder weitreichenden, prinzipiell aber gegebenen Parteienregie jedoch unverzichtbar. Andernfalls könnte man sich gleich für die Anrufung eines Gerichtshofs entscheiden.

Das Spektrum der dargestellten Varianten bietet den Parteien jedenfalls die Wahl, im gegenseitigen Einvernehmen eine mehr oder minder große Portion ihrer einzelstaatlichen Kompetenzen zur Disposition zu stellen, stets unter Wahrung der Chance, im Verfahren zu einem Ergebnis zu gelangen.

Was die persönliche Qualifikation und spezifische Expertise der Schiedsrichter betrifft, so besteht kein Zwang zum »Juristenmonopol« wie bei Gerichtshöfen. Es können vielmehr auch Personen ernannt werden, die über sonstige Kenntnisse verfügen, die sie zur Beurteilung einer Streitsubstanz besonders geeignet erscheinen lassen. Ein Schiedsspruch nach Völkerrecht ist von ihnen allerdings nur eingeschränkt zu erwarten. Um (völker-)rechtliche Gesichtspunkte aber auch in diesen Fällen nicht gänzlich ausblenden zu müssen, wäre dem Kollegium ein juristischer Beraterstab zur Seite zu stellen oder Juristen als Beisitzer ohne Stimmrecht in das Gremium aufzunehmen. Gangbar wäre aber auch der Weg, Juristen und Nichtjuristen gleichzeitig als Schiedsrichter zu bestellen. Wenn das Kollegium jedoch nur aus Juristen gebildet werden sollte, wären ihnen - vice versa - je nach Konfliktmaterie einschlägige Experten als Berater bzw. Beisitzer zur Verfügung zu stellen, so weit die Anhörung von geladenen Sachverständigen während des Verfahrens nicht ausreichen würde.

Auch der soeben beschriebene Vorzug spricht in entsprechend gelagerten Fällen für die Wahl eines Schiedsgerichts: Die Richterbank eines internationalen Gerichtshofs ist aus der immanenten Logik dieser Form der Gerichtsbarkeit heraus nur mit Juristen besetzbar.
2. Die Verfahrensordnung wie das anzuwendende Recht können ebenfalls von den Streitparteien bestimmt werden, soweit nicht gegen die Grundregeln eines fairen Verfahrens oder zwingendes Völkerrecht (ius cogens) verstoßen wird.

Diese genuine Flexibilität gestattet - in den aufgezeigten Grenzen - auch die Abweichung von strikten Völkerrechtsnormen zugunsten anderer Rechtsordnungen oder des Prinzips der Billigkeit als der Gerechtigkeit des Einzelfalles. Ein solches Urteil »ex aequo et bono « kann mit Zustimmung der Parteien - sogar ein zwischen ihnen bestehendes Rechtsverhältnis aufheben oder abändern, darf also »contra legem « erfolgen und daher auch als Instrument des politisch inspirierten "peaceful change « genutzt werden. (Billigkeitsaspekte »intra legem « werden auch vom IGH als Hilfe zur Auslegung und Anwendung geltender Völkerrechtsnormen herangezogen, insbesondere bei der Abgrenzung von Seegebieten. Billigkeitsregeln »praeter legem « können der Schließung von Lücken im Völkerrecht dienen.)

Als problematisch gilt aber die Tatsache, dass ein auf dieser Basis ergehender Urteilsspruch kaum vorhersehbar ist, so dass Streitparteien vor diesem schwer kalkulierbaren Risiko zurückschrecken. Ein Indiz für die Richtigkeit dieser Aussage bildet die Tatsache, dass weder der Ständige Internationale Gerichtshof der Völkerbundära noch der IGH von den Streitparteien jemals ermächtigt wurden, ein Urteil »ex aequo et bono« zu fällen.

Als Rückversicherung wäre alternativ die Wahl der »Allgemeinen Rechtsgrundsätze ", als Rechtsquelle auch im IGH-Statut anerkannt, als anzuwendende Rechtsgrundlage empfehlenswert. Diese Grundsätze heben sich als »general principles of law von den »general principles of justice « des »ex aequo et bono«-Ansatzes $\mathrm{ab}$ und sind daher in jedem Falle »intra legem « angesiedelt.

Eine weitere Variante, die auch vor Gerichten angewandt werden kann, besteht darin, dass auf Wunsch der Parteien nicht ein abschließendes präzises Urteil gefällt wird, sondern lediglich die Grundsätze und Regeln festgestellt werden, nach denen sich die Parteien im Rahmen einer anschließenden bilateralen Einigung auf Vertragsbasis $\mathrm{zu}$ richten haben. Insofern sehen sich die Kontrahenten nicht mit einer endgültigen Entscheidung einer Drittpartei konfrontiert, sondern behalten eine zwar eingeschränkte, prinzipiell aber noch vorhandene Entscheidungsfreiheit bei.

Ein anderer souveränitätswahrender Weg ist mittels Nutzung der »advisory arbitration « gangbar, in deren Rahmen nicht um ein Urteil ersucht wird, sondern um ein per se unverbindliches Rechtsgutachten. Die Streitparteien verpflichten sich jedoch vorab, angesichts der dann gegebenen Kenntnis der Rechtslage zu einer ihr gerecht werdenden bilateralen Einigung zu finden. (Es steht den Parteien allerdings auch frei, ein Rechtsgutachten a priori als verbindlich anzuerkennen; der Unterschied zu einem Urteilsspruch bestünde dann aber lediglich u.a. im Verfahrensablauf.) 
3. Das Schiedsverfahren findet unter Ausschluss der Öffentlichkeit statt. Höchstens der Schiedsspruch wird publik gemacht, kann aber ebenso gut vertraulich bleiben. Die Parteien können somit jedes unwillkommene Aufsehen vermeiden und müssen keinen »Gesichtsverlust« befürchten, wenn ihre Rechtsargumente sich als wenig tragfähig erweisen.

Ferner eröffnet sich den Parteien die flexibilitätssteigernde Option, in Eigenregie einen Schieds-Vergleich in Gestalt eines vereinbarten Schiedsspruchs einzugehen, der nach Zustimmung des Schiedskollegiums die gleiche Bindungswirkung entfaltet wie ein von ihm autonom erlassener, das Verfahren rechtskräftig abschließender Urteilsspruch.

Die komparativen Vorteile der internationalen Gerichtsbarkeit wiederum liegen - je nach Art des Falles - in der Tatsache der ständigen Existenz des Gerichts wie seiner feststehenden Verfahrensordnung, so dass Streitigkeiten der Parteien über die Zusammensetzung des Spruchkörpers wie seine Vorgehensweise gegenstandslos sind. Zudem weist die Spruchpraxis von Gerichten naturgemäß eine höhere Kontinuität und Konsistenz auf. Schließlich kann die Institution über besonderes Ansehen verfügen, was nicht zuletzt der Autorität ihrer Entscheidungen zu Gute kommt.

Optimierte Modelle der Schiedsgerichtsbarkeit und Gerichtsbarkeit weisen eine Reihe unverzichtbarer Funktionserfordernisse auf. Diese sind neben der breiten Parteifähigkeit, der Einlassungspflicht (Obligatorium) und der gesicherten Vollstreckung von Gerichtsurteilen die folgenden:

1. Die Bildung des Spruchkörpers ist - soweit nicht bereits erfolgt - unter allen Umständen zu garantieren. Im Falle der Schiedsgerichtsbarkeit sollte nicht nur dieses Rechtsinstitut als solches institutionell und obligatorisch verankert sein (d.h. die Pflicht, einer einseitigen Klageerhebung Folge zu leisten und an der Bildung eines Spruchkörpers teilzunehmen). Vielmehr sollte auch das konkrete Tribunal bereits gebildet sein, indem die Schiedsrichter als seine Mitglieder schon vorab ernannt worden sind.

Im Falle eines Obstruktionsversuchs der beklagten Partei oder sonstiger Einigungshindernisse muss prophylaktisch die subsidiäre Ernennung der fehlenden Richter durch eine Drittpartei a priori gesichert sein. Als ernennende Stelle kommen z.B. der Präsident des IGH oder der Generalsekretär des PCA bzw. der Vereinten Nationen in Frage, in Seerechtsangelegenheiten auch der Präsident des Internationalen Seegerichtshofs. Für religiös orientierte Parteien römisch-katholischer Observanz schiene auch der Papst geeignet, dessen Autorität etwa in lateinamerikanischen Ländern sich z.B. in der erfolgreichen Vermittlungstätigkeit im hochbrisanten Beagle-Kanal-Streit zwischen Argentinien und Chile offenbarte.

Wenn die ernennende Instanz den Parteien, die sich auf die einvernehmliche Bestellung eines Oberschiedsrichters bzw. Schiedsgerichtsvorsitzenden nicht einigen können, entgegenkommen möchte, so kann sie eine Liste mit Namen potenzieller Richter erstellen und sie den Parteien übermitteln. Diese können sich sodann darüber äußern, welche Personen ihnen inakzeptabel erscheinen, d.h. einen Teil der Namen streichen. Aus den verbleibenden Personen trifft die ernennende Instanz ihre Wahl.
2. Die Kompetenz-Kompetenz hat beim (Schieds-)Gericht zu liegen. Es befindet selbst über seine Zuständigkeit und die Zulässigkeit der Klage wie über die Geltung etwaiger Vorbehalte, falls diese überhaupt noch vorgesehen sind. Es entscheidet im Zweifelsfall auch über Verfahrensregeln und anzuwendendes Recht, wenn zwischen den Parteien Uneinigkeit darüber besteht.

3. Gerichte wie Schiedsgerichte müssen die Kompetenz zur Anordnung vorsorglicher Maßnahmen zur Sicherung der Rechte der Parteien besitzen, und zwar nicht nur auf Parteienantrag, sondern von Amts wegen. Diese Maßnahmen haben verbindlich zu sein und müssen notfalls durchgesetzt werden können.

4. Einreden dürfen nicht zur Prozessverschleppung genutzt werden können. Sie dürfen daher das Verfahren in der Hauptsache nicht unterbrechen und sind somit erst an seinem Ende zu entscheiden.

5. Das (Schieds-)Gericht muss im Rahmen seiner Untersuchungs- und Ermittlungskompetenz die Befugnis besitzen, Zeugen nicht nur auf Parteienwunsch, sondern kraft Amtes selbstständig zu laden und Untersuchungen (auch vor Ort) durchzuführen bzw. Untersuchungskommissionen zu seiner Unterstützung einzusetzen.

6. Weigert sich eine Partei am Verfahren teilzunehmen, so ist die Kompetenz des Spruchkörpers vorzusehen, ein verbindliches Versäumnisurteil zu fällen.

7. Um den Parteien, die sich der Schiedsgerichtsbarkeit bedienen, eine Absicherung gegen möglicherweise nicht mit der nötigen Korrektheit zustande gekommene Schiedssprüche zu gewährleisten, könnte ein vertraglich vereinbarter Rechtszug zu einer gleichsam »höheren« Instanz, bevorzugt dem IGH, eingerichtet werden. Das Gericht hätte dann die Kompetenz, die Rechtskonformität von Schiedsverfahren und die Gültigkeit des Schiedsspruchs $\mathrm{zu}$ überprüfen, allerdings nicht in inhaltlicher, sondern in formell-rechtlicher Hinsicht. Bei Feststellung von gravierenden Verfahrensmängeln wäre die Annullierung des Spruchs möglich.

8. Um auch die Sachentscheidungen von Schiedsgerichten vollstreckbar zu machen und somit das Parteienvertrauen in die Wirksamkeit dieses Streitbeilegungsinstruments $\mathrm{zu}$ steigern, könnten Vollstreckungsklauseln in die schiedsgerichtlichen Abmachungen aufgenommen werden. Ihre Form könnte die einvernehmliche Ermächtigung des Sicherheitsrats der Vereinten Nationen (bzw. des IGH oder des VN-Generalsekretärs - s.o.) zu Zwangsmaßnahmen sein, wenn der IGH die Rechtmäßigkeit und Rechtskraft eines Schiedsspruchs bestätigt. Die Vollstreckung erfolgt gemäß den oben dargestellten Prozeduren. (Streng genommen fallen die beiden letzten Punkte bereits unter die Rubrik »Komplexmodelle«, die im Folgenden abgehandelt werden.)

\subsection{Komplexe Modelle}

Komplexe Modelle (Verbundmodelle) bauen auf den Basismodellen auf und erweitern sie um zusätzliche Mechanismen gerichtlicher wie außergerichtlicher Art. Eine Variante 
bestünde darin, die Entscheidungen von Schiedsgerichten von einem etablierten Gericht, primär dem IGH, überprüfen $\mathrm{zu}$ lassen und so eine Art Instanzenzug einzurichten (s.o.). Dies würde zum einen das Risiko von schiedsgerichtlichen Kompetenzüberschreitungen minimieren, zum anderen eine Arbitrationsentscheidung bei Bestätigung durch den IGH umso eher vollstreckbar machen.

Komplexitätssteigernd und damit flexibilisierend würde die zwingende oder optionale Vorschaltung außergerichtlicher Mechanismen vor den Gang vor ein (Schieds-)Gericht wirken, indem die Parteien zunächst eine gütliche Einigung über den weitaus weniger konfrontativen Weg des Vergleichs suchen, sofern nicht bereits die Einsetzung einer Untersuchungskommission durch Klärung der Fakten zur Beendigung des Disputs hinreicht. Die Nutzung einer Vergleichskommission kann den Parteien den Gang vor Gericht ersparen, indem sie eine einvernehmliche Lösung des Streitfalls herbeiführt. Der Rechtsweg stünde aber weiterhin offen, sobald die Vergleichskommission das Scheitern ihrer Schlichtungsbemühungen feststellt.

Damit ist es aber nicht getan. Auch das Schlichtungsoder Vergleichskonzept bedarf der Optimierung, um gewisse Schwächen zu kompensieren und seine spezifischen Stärken weiter auszubauen.

Zunächst ist festzuhalten, dass der Mechanismus weitaus eher als die (Schieds-)Gerichtsbarkeit geeignet ist, Konflikte zu lösen, die nicht den Charakter eines Rechtsstreits, sondern den eines Regelungskonflikts besitzen, der um die Neugestaltung eines Rechtsverhältnisses und nicht um dessen Feststellung kreist: Eine Vergleichskommission ist prinzipiell nicht an völkerrechtliche Normen gebunden, so weit diese zur Disposition der Parteien stehen, also kein zwingendes Völkerrecht darstellen. Sie kann ihre eo ipso unverbindlichen Vorschläge an den Maßgaben der politischen Zweckmäßigkeit und des billigen Ermessens orientieren und dabei den Positionen der Parteien diplomatisch-flexibel entgegenkommen. Diese selbst suchen mit der Hilfe der Kommission einen Ausgleich ihrer Interessen in Form eines auf dem Wege des gegenseitigen Nachgebens zu erzielenden Kompromisses. Auf diese Weise entfällt das Problem eines potenziellen Nullsummenspiels mit obsiegender und unterliegender Partei, das sich bei gerichtlicher oder schiedsgerichtlicher Entscheidung ergeben kann, wenn diese auf Grund der Rechtslage eindeutig zu Gunsten einer Seite ausfällt. Das Resultat des Verfahrens (gütliche Einigung statt streitige Entscheidung) gestaltet sich somit weniger konfrontativ, wegen der Unverbindlichkeit der Kommissionsvorschläge weniger riskant, und wahrt so die (Prestige-)Interessen beider Parteien jeweils zumindest zum Teil. Hierzu trägt auch seine absolute Vertraulichkeit bei, die für die Empfehlungen der Kommission gleichermaßen gilt.

Die Charakteristika dieses besonders souveränitätsfreundlichen Institutes stellen sich im Detail wie folgt dar: Da keine strikte Bindung an Rechtsvorgaben besteht, kann die Kommission von den Parteien aus Diplomaten oder Experten der verschiedensten Sachgebiete zusammengestellt werden. Dies könnte analog den obigen Mechanismen der Bildung von Schiedsgerichten erfolgen, so dass die Schaffung der Kommission in jedem Falle gewährleistet wäre. $\mathrm{Zu}$ bevorzugen wären auch hier »stehende«, d.h. vorab installierte und ständig einsatzbereite Gremien.
Die Kommission ist in jedem Falle gehalten, die Grundsätze der Verfahrensgerechtigkeit zu beachten. Das Schlichtungsverfahren selbst vereint die Elemente von Untersuchung und Vermittlung (das Kollegium wird daher auch als Vermittlungskommission bezeichnet), ist im Gegensatz zur klassischen Mediation aber schiedsgerichtsähnlich formalisiert. (Der Form zum Trotz ist seine Funktion aber eine außergerichtlich-diplomatische.) In diesem Kontext sind die Befugnisse der Kommission zur Tatsachenfeststellung so effektiv wie möglich auszugestalten. Dazu gehören vor allem auch die Kompetenz zur Einbestellung von Zeugen aus eigener Initiative, d.h. von Amts wegen, und - wo erforderlich - die ungehinderte Durchführung von Inspektionen vor Ort.

Die Gewichtsverteilung zwischen den Komponenten »Untersuchung « und »Vermittlung « im Rahmen eines Vergleichsverfahrens sollte von den Parteien einvernehmlich ihren Bedürfnissen und dem Konfliktprofil gemäß bestimmt werden können, je nachdem, ob es eher auf Faktenermittlung oder auf einen kreativen Lösungsvorschlag ankommt. (Insofern kann eine Vergleichskommission tendenziell auch die Gestalt einer Untersuchungskommissionannehmen bzw. deren Funktion erfüllen.) Im Allgemeinen wird aber die zweite Variante bevorzugt werden, da es letztlich primär um die Erzielung eines streitbeilegenden Kompromisses geht. Zu diesem Zweck ist der Schlichtungskommission ein maximaler Handlungsspielraum einzuräumen. Sie muss z.B. die Möglichkeit haben, mit den Parteien auch jeweils einzeln zu kommunizieren, um deren Positionen besser verstehen und ihre Empfehlungen darauf abstimmen zu können. Ebenso muss es ihr gestattet sein, »package deals« vorzuschlagen, die Zugeständnisse einer Partei, die den eigentlichen Streitgegenstand betreffen, durch Kompensationen ausgleichen, die einem Sachbereich außerhalb der aktuellen Streitsubstanz entstammen. Vergleichsvorschläge der Kommission sind zunächst als eher informell zu betrachten, damit bei Nichtannahme durch die Parteien das Verfahren nicht als gescheitert betrachtet werden kann und einem neuen, modifizierten Vorschlag nichts im Wege steht. Der Vorgang sollte wiederholbar sein, solange die Kommission noch an Erfolgsaussichten glaubt. Andererseits müsste es der Kommission freistehen, einem solchen Vorschlag formellen Charakter zu verleihen, wenn sich eine Parteieneinigung auf seiner Basis konkret abzeichnet. Den Parteien selbst wäre es aber auch unbenommen, einen Vorschlag einvernehmlich in einer von ihnen abgeänderten Form anzunehmen (wie in jedem Falle üblich völkerrechtlich verbindlich in Gestalt eines bilateralen Vertrags, womit der Unverbindlichkeitscharakter der ursprünglichen Empfehlung der Bindungswirkung der Einigungsformel weicht).

Eine andere Variante eröffnet sich, wenn die Parteien eine formelle Vergleichsempfehlung vorab gegenseitig als verbindlich anerkennen. Eine Identität mit dem Spruch eines Schiedsgerichts würde auch unter diesen Prämissen nicht hergestellt, da der Inhalt der verbindlichen Formel ja auf gänzlich anderem Wege zustande käme.

Neben diesen Vorzügen des Schlichtungsmodells seien auch seine Schwächen nicht verschwiegen:

1. Ein etwaiges Machtgefälle zwischen den Parteien ist im Rahmen dieses Verfahrens durch die Kommission nicht kompensierbar. Ihre Empfehlungen sind unverbindlich und können eine Partei, die sich ein Fortschwelen eines 
Konfliktes leisten kann, nicht zum Einlenken bewegen, es sei denn unter Erfüllung ihrer Forderungen, die einseitig zu Lasten der anderen Partei gehen können.

2. Prinzipiell ist ein streitabschließendes Resultat nicht garantiert, da dieses allein von der Kompromissbereitschaft der Parteien abhängt.

Schon aus diesem Grund sind weitere Mechanismen in Gestalt der (Schieds-)Gerichtsbarkeit erforderlich, die nachfolgend aktiviert werden können und diesen Defiziten, die sich in bestimmten Fällen aktualisieren, abhelfen. In diesem »Instanzenzug « gebührt dem Vergleichsverfahren aber sein bedeutender Rang als Einstiegsoption in den Prozess der friedlichen Streitbeilegung, die aber auch einen Eigenwert aufweist, wenn sie selbst (als Güteverfahren) schon zum Erfolg führt.

Unter Einbeziehung des Schlichtungsmodells sind (s.o.) im Zuge einer Ausdifferenzierung des Streiterledigungssystems diverse Kombinationen von komplementären Mechanismen vorstellbar: Rechtsprechung, d.h. Streitbeilegung durch ebenso autoritative wie bindende gerichtliche bzw. schiedsgerichtliche Entscheidungen, und eher diplomatische, dennoch aber formalisierte Methoden i.S.v. Schlichtung/Vergleich. Diese Modelle unterscheiden sich hinsichtlich ihrer Charakteristik, Reichweite oder Verbindlichkeit; sie können flexibel der jeweiligen Konfliktlage und den Konfliktbearbeitungsbedürfnissen angepasst werden. Dazu sind diverse Kombinationen dieser einzelnen Bausteine möglich, insbesondere in dem Sinne, dass bei ungewöhnlich komplizierten und verfahrenen Streitfällen verschiedene Prozeduren hintereinandergeschaltet werden können, bis eine allseits akzeptable Lösung erreicht ist. Diese Phasen-, Integrativ- oder Verbundmodelle implizieren gerichtliche, schiedsgerichtliche und außergerichtliche Streitschlichtungs- und Streiterledigungsmechanismen, Module, die im Verein eine endgültige Streitentscheidung garantieren und so den Rechtsfrieden zwischen den Streitparteien nachhaltig wieder herstellen sollen.

Ein weiteres relevantes Charakteristikum dieser optimierten Modellvarianten besteht darin, dass sie unterschiedliche Anforderungen an die Reformbereitschaft der Staatenwelt stellen, also den Nationalstaaten mehr oder minder große Souveränitätsopfer abverlangen. Damit wird dem gewichtigen Aspekt der politischen Realisierbarkeit von Alternativkonzepten Rechnung getragen: Die souveränitätsbewusste Staatenwelt kann entscheiden, wie weit sie gehen und welches Modell sie (noch) akzeptieren will. Ihr »politischer Wille« zum fortschreitenden Verzicht auf den Fetisch Souveränität könnte von ihren Zivilgesellschaften allerdings nach Kräften gefördert werden.

Die einzelnen Ablaufmechanismen lassen sich am plastischsten anhand der Abbildung 2 erläutern:

Die erste Option besteht in der Wahl zwischen den Instrumenten Vergleichskommission, Schiedsgericht und Gericht. Die zweite Option setzt beim Vergleich an und endet mit dieser Instanz im Falle einer erfolgreichen Schlichtung. Bei einem Fehlschlag steht der Weg zur Schiedsgerichtsbarkeit oder Gerichtsbarkeit offen. Dort angelangt, kann sich unter bestimmten Umständen die Notwendigkeit ergeben, einen weiteren Schritt einzuleiten. Er führt von der Schiedsgerichtsbarkeit zu einem etablierten internationalen Gericht, wenn die Bildung eines eigentlich vorgesehenen Schiedsgerichts nicht zustande kommt (z.B. wegen Regelungsmängeln im Schiedsvertrag), wenn ein ergangener Schiedsspruch auf seine Gültigkeit (bzw. Nichtigkeit) überprüft werden soll, oder wenn seine zwangsweise Vollstreckung beantragt werden soll. Bei diesem Gericht wird es sich in der Regel um den IGH handeln.

\section{Abb. 2: Ablaufdiagramm: Optionen der Streiterledigung}

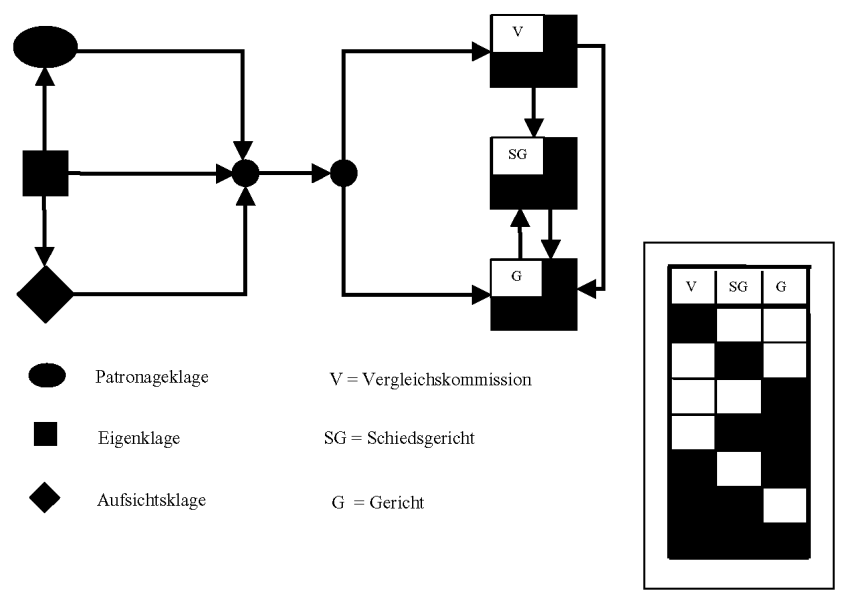

Vice versa kann von der zunächst vorgesehenen Gerichtsbarkeit zur Bildung eines Schiedsgerichts übergegangen werden, wenn das angerufene Gericht sich weigert, den Fall zu verhandeln, da es wider das Erwarten der Parteien unüberwindbare Verfahrenshindernisse konstatiert. Die einzelnen Institute der friedlichen Streitbeilegung können mithin alternativ ausgewählt werden (Basismodelle) oder sukzessiv bzw. kumulativ - in verschiedenen Kombinationen und auf unterschiedlichen Pfaden - hintereinander geschaltet werden (komplexe Modelle). Im zweiten Fall kann die nächst folgende Instanz insbesondere im Sinne der Subsidiarität tätig werden, wenn die vorhergehende Instanz die Angelegenheit nicht regeln konnte. (Die Kombinationsmöglichkeiten sind in Abb. 2 auch in abgewandelter grafischer Form in einem Kasten dargestellt.)

\section{Resümee}

Das hier präsentierte System der friedlichen Streitbeilegung sollte im Stande sein, durch die Behebung der wesentlichen Defizite der bisherigen Konzepte eine verlässliche Gewähr dafür zu bieten, dass internationale Konflikte und Streitigkeiten ohne Rückgriff auf Drohung und Gewalt auf zivilisierte Weise ausgetragen werden können. Die »immoral minority «, die genau weiß, dass ihre machtpolitischen Ziele jeder Rechtsgrundlage entbehren und dass dieser friedliche Weg für sie deshalb nicht von Erfolg gekrönt sein kann, kann vom Appell an die Waffen jedoch erst dann abgehalten werden, wenn die Vereinten Nationen endlich in die Lage versetzt werden, ihre Friedenssicherungsfunktion effektiv zu erfüllen. So lange dies nicht der Fall ist, so lange also kein Zwang zur ausschließlichen Wahl des Rechtsweges besteht und die Vollstreckung von Urteilen nicht garantiert ist, wird die internationale (Schieds-)Gerichtsbarkeit bei aller Perfektionierung und Ausdifferenzierung ihres Regelwerks an ihre Grenzen stoßen, die sie aus eigener Kraft nicht 
überwinden kann. Des ungeachtet erfüllt sie aber auch in diesen Grenzen eine unverzichtbare Funktion zur Wahrung von Frieden und Recht. Sie kann dieser Aufgabe umso eher gerecht werden, je zuverlässiger ihre Regelungsdefizite eliminiert sind und je weiter die Pflicht der Staatenwelt zur Nutzung dieser Instrumente entwickelt ist.

Kathryn Tätzsch

\section{Konflikte im Kontext der indonesischen Innen- und Sicherheitspolitik und der weltpolitischen Großwetterlage}

\author{
Kathryn Tätzsch, M.A., World \\ Vision Germany, Internatio- \\ nal Programmes/Humanitarian \\ Assistance, Friedrichsdorf
}

Die Aufmerksamkeit der politischen Weltöffentlichkeitrichtete sich nach den Bombenanschlägen auf der Ferieninsel Bali am 12. Oktober 2002 auf Indonesien, denn der Schock über die knapp 200 Toten und die vielen Verletzten - v.a. Urlauber - und das Ausmaß der Zerstörung erinnerten teilweise an die Attentate des 11. September 2001 in den USA. Es stellt sich die Frage, vor welchem sicherheitspolitischen, sozialen und ökonomischen Hintergrund diese Bombenattentate ihre Wirkung gezeigt haben bzw. wie sie damit verwoben sind.

\section{Indonesien - Jahre des Umbruchs}

Das autoritäre Regime unter dem indonesischen Staatspräsidenten Suharto, welches als »New Order « insbesondere einen Block aus dem indonesischen Militär (Tentara Nasional Indonesia - TNI), bürokratischen Eliten und einflussreichen Wirtschaftsvertretern bildete, basierte weitgehend auf patrimonialer Symbiose. ${ }^{1}$ Nach Einsetzen der ökonomischen Krise in Südostasien, die auch für die Wirtschaft in Indonesien ab der zweiten Hälfte des Jahres 1997 dramatische Ausmaße annahm, setzten die immer lautstärkeren Proteste, zunächst von Studierenden in den größeren Städten der Inseln Java und Sulawesi, die Regierung in Jakarta unter Druck. Später lehnten sich in Demonstrationen auch Universitätsangestellte und weitere Berufsgruppen zunächst gegen Preiserhöhungen, dann direkt gegen die politische Führung auf. Diese Proteste richteten sich nachhaltig gegen Korruption und Nepotismus der Suharto-Regierung, trugen jedoch auch anarchische und rassistische Züge. Es kam zu Ausschreitungen und Plünderungen durch einige Demonstranten, zuweilen zu Lynchmorden, auch gegen die chinesische Minderheit, meist wohlhabende Geschäftsleute. Dies und die drohende Gefahr einer Staatsimplosion erzeugte die unverhältnismäßige gewalttätige Reaktion von staatlichen Sicherheitskräften mit ca. 1.000 To-

1 Vgl. Ingrid Wessel, The Politics of Violence in New Order Indonesia in the Last Decade of the 20th Century, in: Ingrid Wessel/Georgia Wimhöfer (Hg.), Violence in Indonesia, 2001, S. $103 \mathrm{ff}$. desopfern sowie viele Verletzten. ${ }^{2}$ Schließlich erzwangen die Unruhen Suhartos Rücktritt. Dessen Nachfolger Habibie und seinem Reformkabinett wurde allerdings bald vorgeworfen, die selbe Politik unter einem neuen »Etikett« fortzuführen.

Tatsächlich wurde er bereits im Herbst 1999 von Abdurraham Wahid im Präsidentenamt abgelöst, der die einflussreiche politische Rolle des Militärs zum ersten Mal beschränkte. Megawati Sukarnoputri, die Tochter des ersten indonesischen Präsidenten, wurde im August 2001 Präsidentin. $^{3}$

Für die nach dem Ende der repressiven Suharto-Ära immer stärker zutage tretenden Konflikte in dem aus über 13.000 Inseln und mehr als 206 Millionen Einwohnern ${ }^{4}$ und ca. 250 unterschiedlichen Sprachen bestehenden multiethnischen ${ }^{5}$ Staat lässt sich nicht nur die asiatische Wirtschaftskrise Ende der 90er Jahre verantwortlich machen. Auch können nicht die plötzlich freigesetzten, bereits seit Jahrzehnten unter der autoritären politischen Führung mittels staatlicher Sicherheitskräfte opprimierten Konfliktkonstellationen für die Gewalteruptionen verantwortlich gemacht werden. Häufig, aber nie ausschließlich, hatten diese Konflikte ihre Wurzeln in ethnischer oder religiöser Gruppenzuschreibung. Diese allein können aber nicht hinreichend zur Erklärung für die Mannigfaltigkeit der Kämpfe, Bombenanschläge, Morde, Plünderungen, Vertreibungen und Massaker herangezogen werden. Oftmals, insbesondere seit dem Attentat am 11. September 2001 in den USA, werden Konflikte, auch in Indonesien, von internationalen Medien vorschnell als ausschließliche Religionskonflikte (zwischen Islam - oft in Verbindung zu militantem Islamismus - und Christentum) dargestellt. Es

2 Vgl. Georgia Wimhöfer, Indonesian Students in 1998. Civil Society and the effects of violence, in: Wessel/Wimhöfer (Hg.), Violence in Indonesia, 2001, S. 169.

3 Vgl. Freedom House - Country and Territory Reports Indonesia 2002, http://freedomhouse.org/research/freeworld/2002/countryratings/indonesia.htm.

4 Vgl. ebda.

5 Ethnie soll hier mit folgenden minimalen Wesensmerkmalen definiert werden: Sie ist eine historisch gewachsene Gemeinschaft, mit spezifischer Kultur und speziellen kollektiven historischen Gedächtnis(-mythen) über gemeinsame Abstammung etc., einschließlich eines immer von Neuem reproduzierten Solidaritätsgefühls. Vgl. Christian P. Scherrer, EthnoNationalismus im Weltsystem. Prävention, Konfliktbearbeitung und die Rolle der internationalen Gemeinschaft. Agenda Verlag, Münster 1996, S. $15 \mathrm{f}$. 